\title{
LA PROTECCIÓN DE LA CALIDAD DEL AIRE ANTE LOS TRIBUNALES DE JUSTICIA EN UN CONTEXTO DE LUCHA CONTRA EL CAMBIO CLIMÁTICO
}

\section{THE PROTECTION OF AIR QUALITY STANDARDS BEFORE COURTS OF JUSTICE IN A CONTEXT OF THE FIGHT AGAINST CLIMATE CHANGE}

\author{
JAIME DORESTE HERNÁNDEZ \\ Abogado ambientalista - Magerit Abogados \\ Profesor Asociado de Derecho Ambiental - Universidad Autónoma de Madrid. \\ jaimedoreste@icam.es
}

Fecha de recepción: 10 de abril de 2020 / Fecha de aceptación: 7 de julio de 2020

RESUMEN: Precedida y avalada por la experiencia de las organizaciones ambientalistas en la protección jurisdiccional de la calidad del aire, recientemente se ha entablado en España un primer litigio climático. Sus promotores lo han formulado como un instrumento para exigir una mayor y más decidida acción pública en materia de mitigación climática alineada con las recomendaciones científicas y que garantice además los derechos humanos y el derecho a un medio ambiente adecuado de las generaciones presentes y futuras.

RESUM: Precedit i avalat per l'experiència de les organitzacions ambientalistes en la protecció jurisdiccional de la qualitat de l'aire, recentment s'ha entaulat a Espanya un primer litigi climàtic. Els seus promotors I'han formulat com un instrument per exigir una major i més decidida acció pública en matèria de mitigació climàtica alineada amb les recomanacions científiques i que 
garanteixi, a més, els drets humans i el dret a un medi ambient adequat de les generacions presents i futures.

ABSTRACT: Preceded and supported by the experience on proceedings regarding the protection of air quality standards by environmental organisations, the first climate litigation has recently been filed in Spain. Its promoters have formulated it as an instrument to defend greater and more determinated public action on climate mitigation. This must be in line with scientific recommendations and should also guarantee human rights and the right to an adequate environment for present and future generations.

PALABRAS CLAVE: Litigación climática - Contaminación atmosférica Calidad del aire - Cambio climático - Acceso a la justicia

PARAULES CLAU: Litigació climàtica - Contaminació atmosfèrica - Qualitat de l'aire - Canvi climàtic - Accés a la justícia

KEYWORDS: Climate Litigation - Atmospheric pollution - Air quality - Climate Change - Access to justice

SUMARIO: I. LA IMPORTANCIA DEL ACCESO A LA JUSTICIA EN LA LUCHA CONTRA EL CAMBIO CLIMÁTICO. II. APROXIMACIÓN AL 'LITIGIO CLIMÁTICO’. III. LITIGIOSIDAD EN MATERIA DE CALIDAD DEL

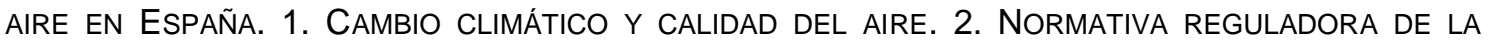
CALIDAD DEL AIRE Y LA CONTAMINACIÓN ATMOSFÉRICA. 3. LA CRECIENTE LITIGIOSIDAD EN MATERIA DE CAlidad del aire. 3 A) Planes y Estrategias de Calidad del Aire. 3 B) LA ZBE 'Madrid Central'. IV. La Litigiosidad Climática en España. V. A mOdo de CONClusión y PeRspectivas DE FUTURO. VI. BIBLIOGRAFÍA. 


\title{
I. LA IMPORTANCIA DEL ACCESO A LA JUSTICIA EN LA LUCHA CONTRA EL CAMBIO CLIMÁTICO.
}

\begin{abstract}
"Somos la primera generación que entiende perfectamente lo que está pasando con el clima y posiblemente seremos la última que pueda evitar la catástrofe hacia la que nos dirigimos" Jorge Riechmann
\end{abstract}

Decía JOHN KNOX, ex relator especial de las Naciones Unidas sobre los derechos humanos y el medio ambiente, que "El cambio climático pone en peligro el disfrute de un amplio abanico de derechos humanos. A la inversa, para poder combatirlo eficazmente es necesario ejercer esos derechos, entre ellos los derechos a la información y la participación"1. Cierto, pero en nuestra opinión se olvidaba de uno, del derecho de acceso a la justicia, consagrado en la Constitución Española como el derecho a la tutela judicial efectiva.

Porque es precisamente el derecho de acceso a la Justicia la más eficaz herramienta para garantizar o coadyuvar al cumplimiento de las normas ambientales $^{2}$. Por su carácter de clausula de cierre para la protección y garantía de los derechos de participación y transparencia ambiental y, en suma, del derecho ambiental, el Derecho Internacional, a través del Principio $10^{\circ}$ de la Cumbre de Río ${ }^{3}$-desarrollado por el Convenio de Aarhus ${ }^{4}$ y el

\footnotetext{
1 KNOX, J en "Cambio climático y derechos humanos", Observatorio Social de la Caixa, septiembre de 2016, disponible en: <https://observatoriosociallacaixa.org/-/cambio-climatico-yderechos-humanos>.

2 SANCHIS MORENO, F. "Convenio de Aarhus; Guía sobre el Acceso a la Justicia Ambiental", Consejo General de la Abogacía Española-Asociación para la justicia ambiental (ELAW España) 2007.

${ }^{3}$ La Declaración de la Conferencia de Naciones Unidas sobre Medio Ambiente y Desarrollo, celebrada en Río de Janeiro en junio de 1992, recoge 27 principios, generalmente aceptados como los principios básicos del derecho ambiental. El décimo reza:
}

"El mejor modo de tratar las cuestiones ambientales es con la participación de todos los ciudadanos interesados, en el nivel que corresponda. En el plano nacional, toda persona deberá tener acceso adecuado a la información sobre el medio ambiente de que dispongan las autoridades públicas, incluida la información sobre los materiales y las actividades que encierran peligro en sus comunidades, así como la oportunidad de participar en los procesos de adopción de decisiones. Los Estados deberán facilitar y fomentar la sensibilización y la participación de la población poniendo la información a disposición de todos. Deberá proporcionarse acceso 
Acuerdo de Escazú5-, ha puesto de relieve la importancia y necesidad del acceso a la justicia en materia ambiental ${ }^{6}$.

En términos ahora de derecho interno, el Tribunal Constitucional tiene establecido que el derecho a la tutela judicial efectiva que reconoce el artículo 24.1 de la Constitución Española se erige como una garantía frente a la arbitrariedad e irrazonabilidad de los poderes públicos (SSTC 147/1999, 87/2000, 221/2001, 55/2003, 223/2005, 177/2007, 134/2008, 191/2011 у 8/2014, entre otras muchas). En Estados Democráticos de Derecho, un sistema de justicia o poder judicial independiente de los poderes legislativos y ejecutivo se configura como un contrapoder que, por una parte, controlan el ejercicio de las potestades de gobierno y ejecutivas y, por otra, garantizan el cumplimiento de la Ley. La propia Constitución Española en su artículo 106, hace uso de ese alcance general cuando establece que los Tribunales controlan "la legalidad de la actuación administrativa, así como el sometimiento de ésta a los fines que la justifican".

En este sentido cobra especial relevancia la jurisdicción contenciosoadministrativa, a la que compete la revisión de la actividad (e inactividad) de la Actuación. A este respecto, no podemos dejar de destacar las palabras del Preámbulo de la Ley 29/1998, de 13 de julio, reguladora de la Jurisdicción Contencioso-administrativa cuando señalan que:

efectivo a los procedimientos judiciales y administrativos, entre éstos el resarcimiento de daños y los recursos pertinentes."

${ }^{4}$ Convenio de la Comisión Económica para Europa de Naciones Unidas sobre acceso a la información, la participación del público en la toma de decisiones y el acceso a la justicia en materia de medio ambiente, hecho en Aarhus el 25 de junio de 1998.

Fue ratificado por España el 28 de diciembre de 2004 y su instrumento de ratificación se publicó en el BOE núm. 40 de 16 de febrero de 2005.

${ }^{5}$ Acuerdo Regional sobre el Acceso a la Información, la Participación Pública y el Acceso a la Justicia en Asuntos Ambientales en América Latina y el Caribe, adoptado en Escazú (Costa Rica) el 24 de marzo de 2018.

${ }^{6}$ Para SALAZAR, el acceso a la justicia ambiental "es la vertiente más transformadora del [Convenio de Aarhus] pues no sólo garantiza el acceso a la información ambiental y que la participación sea real y efectiva, sino que supone la posibilidad de que ciudadanos y sus organizaciones exijan la aplicación del Derecho ambiental (artículo 9.3) y se eviten así los daños al ecosistema y los peligros para la salud".

Vid. SALAZAR ORTUÑO, E. "El acceso a la justicia ambiental, una cuestión pendiente del Convenio de Aarhus", 2019, CGAE, accesible en:

$<$ https://www2.abogacia.es/publicaciones/blogs/blog-derecho-ambiental/el-acceso-a-la-justiciaambiental-una-cuestion-pendiente-del-convenio-de-aaerhus/>. 
"Lo que realmente importa y lo que justifica la existencia de la propia Jurisdicción Contencioso-administrativa es asegurar, en beneficio de los interesados y del interés general, el exacto sometimiento de la Administración al derecho en todas las actuaciones que realiza en su condición de poder público y en uso de las prerrogativas que como tal le corresponde".

Recordemos que de conformidad con lo dispuesto en el artículo 45.2 de nuestro texto constitucional, corresponde a los poderes públicos velar "por la utilización racional de todos los recursos naturales, con el fin de proteger $y$ mejorar la calidad de la vida y defender y restaurar el medio ambiente, apoyándose en la indispensable solidaridad colectiva", como reverso positivo del derecho a disfrutar de un medio ambiente adecuado para el desarrollo de la persona que consagra el apartado primero de dicho artículo.

Es por todo ello que no resulta excesivo asegurar que la garantía del cumplimiento del derecho ambiental (y dentro de éste, de los Convenios y Tratados Internacionales relativos a la lucha contra el Cambio Climático) por parte de los poderes públicos, y singularmente del Ejecutivo, formaría parte de la esencia misma de la jurisdicción contencioso-administrativa, sin perjuicio de las dificultades tanto materiales como formales que en nuestro ordenamiento jurídico presenta el litigio ambiental ${ }^{7}$.

A nivel internacional, en todo caso, la litigación climática se está convirtiendo en una vía bastante efectiva para presionar a los Estados e incidir en sus políticas climáticas, precisamente porque surgen del fracaso de la política frente a los enormes retos de nuestros tiempos ${ }^{8}$.

Buen ejemplo del interés e importancia de esta litigación climática es la atención que mereció con motivo de la celebración de la COP 25 en Madrid, en side events como los organizados la Iniciativa de Derecho Climático y Gobernanza en la Facultad de Derecho de la Universidad Autónoma de

\footnotetext{
${ }^{7}$ Así lo han puesto de relieve numerosas autoras, entre ellas FERNÁNDEZ EGEA, R y SIMOU, S en "Litigación climática en España: posibilidades y límites", Revista de Derecho Urbanístico y medio ambiente núm. 328, marzo 2019 o VILASECA BOIXAREU, I. y SERRA CALVÓ, J, en "Litigación climática y separación de poderes", Revista Catalana de Dret Ambiental Vol. IX núm.2 (2018).

8 Vid. VILASECA BOIXAREU, I. y SERRA CALVÓ, J 2018, ope. cit.
} 
Madrid9, o por la Universidad de Murcia en colaboración con el Instituto Pascual Madoz de la Universidad Carlos III denominado "Litigios Climáticos y Justicia"10, la sesión organizada por la Sección de Derecho ambiental del Colegio de la Abogacía de Barcelona 'La litigación climática: situación actual y retos de futuro'11, el I Congreso Ítalo-Español sobre la lucha en clave judicial frente al cambio climático organizado por la Universitat Jaume I los días 19 y 20 de noviembre $2020^{12}$, 'La litigación climática: situación actual y retos de futuro' o la multitud de estudios y artículos doctrinales publicados al respecto en esta revista ${ }^{13}$.

\section{APROXIMACIÓN AL 'LITIGIO CLIMÁTICO’}

Por supuesto, no todo pleito o procedimiento en el que sea de aplicación el corpus jurídico relativo a la contaminación atmosférica o a los instrumentos de lucha contra el Cambio Climático puede, en puridad, considerarse "litigación climática". Entendemos, como hacen FERNÁNDEZ EGEA, R y SIMOU, $\mathrm{S}^{14}$, que al referirnos a la Litigación Climática hablamos de procedimientos judiciales en los que organizaciones sociales o ambientales, y aún particulares, actúan contra la inactividad administrativa en la lucha contra el cambio

\footnotetext{
${ }^{9}$ El "Climate Law and Governance Day 2019", cuyo programa puede consultarse en: $<$ http://www.climatelawgovernance.org/events/climate-law-governance-day-2019-2/>.
}

10 Cuyo programa puede consultarse en: <http://www.institutopascualmadoz.es/wpcontent/uploads/2019/11/litigios-climaticos-10-de-diciembre.pdf $>$.

11 Véase <https://www.icab.es/en/formacion/course/Sesion-virtual-ligitacion-climatica-Lalitigacion-climatica-situacion-actual-y-retos-de-futuro/>

12 Vid. <https://www.actualidadjuridicaambiental.com/agenda-i-congreso-italo-espanol-sobre-lalucha-en-clave-judicial-frente-al-cambio-climatico-universitat-jaume-i-castellon-de-la-plana-19-y20-de-noviembre-2020/>

Puede consultarse el programa en $<$ https://redhexata.com/i-congreso-italo-espanol-sobre-lalucha-en-clave-judicial-frente-al-cambio-climatico/>.

${ }^{13}$ A modo de ejemplo, el Volumen 9, número 2 de 2018 de la Revista Catalana de Dret Ambiental le dedicó al tema nada menos que la Editorial, un Artículo y dos Estudios.

14 FERNÁNDEZ EGEA, R y SIMOU, S, 2019, ope. cit. Las autoras definen aquí 'Litigación Climática' como "actuaciones judiciales llevadas a cabo por particulares o grupos de particulares (pertenecientes a la "sociedad civil») contra la actuación y/o inactividad administrativa en la lucha contra el cambio climático". 
climático o contra la actuación de la Administración que pueda considerarse contraria a ésta ${ }^{15}$.

Desde ésta óptica, no puede reputarse como litigación climática, por ejemplo, los recursos formulados ante el Tribunal Supremo contra el Acuerdo del Consejo de Ministros de 15 de noviembre de 2013, por el que se procede a la asignación, para el período 2013-2020, de los derechos de emisión de gases de efecto invernadero por Repsol Petróleo (rec. 529/2017) ${ }^{16}$ o Siderúrgica Sevillana SA (rec. 439/2017) ${ }^{17}$. Y ello sin perjuicio, por supuesto, de la legitimidad de las pretensiones de las partes, la relevancia de la doctrina jurisprudencial que el asunto pueda sentar o la importancia intrínseca del supuesto (así por ejemplo, en ambos procedimientos y a instancias de la parte actora se formuló cuestión prejudicial comunitaria, resuelta en virtud del Auto del Tribunal de Justicia de la Unión Europea (Sala Sexta) de 26 de octubre de 2016 (Asuntos acumulados C-369/15 a C-372/15). Pero parece claro que la pretensión de los recurrentes en dichos procedimientos no era el incremento de la acción pública en materia de mitigación, sino todo lo contrario: que se les aumentasen los derechos de emisión gratuitos concedidos a las empresas recurrentes en aquel Acuerdo del Consejo de Ministros.

\section{LITIGIOSIDAD EN MATERIA DE CALIDAD DEL AIRE EN ESPAÑA.}

\section{Cambio climático y calidad del aire}

Ciertamente cambio climático y contaminación atmosférica (o calidad del aire, en su vertiente positiva) son dos conceptos diferentes aunque interrelacionados.

\footnotetext{
15 Por el contrario, la definición ofrecida por Markell y Ruh, recogida por VILASECA BOIXAREU, I. y SERRA CALVÓ, J, 2018, ope. cit. - "Any piece of federal, state, tribal, or local administrative or judicial litigation in which the party filings or tribunal decisions directly and expressly raise an issue of fact or law regarding the substance or policy of climate change causes and impacts"- resulta, por una parte, en exceso alambicada, y por otra poco concreta en cuanto al objetivo de la litigación.

${ }^{16}$ Recurso resuelto -y desestimado- en virtud de la Sentencia 529/2017 de la Sección Quinta de la Sala III de 28 de marzo.

17 Recurso resuelto -y desestimado- en virtud de la Sentencia 439/2017 de la Sección Quinta de la Sala III de 13 de marzo.
} 
El cambio climático se produce por la concentración en capas altas de la atmósfera de gases de efecto invernadero (GEI), principalmente por el dióxido de carbono $\left(\mathrm{CO}_{2}\right)$, pero también por otros gases como el metano $\left(\mathrm{CH}_{4}\right)$ o el óxido nitroso $\left(\mathrm{N}_{2} \mathrm{O}\right)$, lo provoca el aumento de la temperatura global del planeta. La Convención Marco de Naciones Unidas sobre el Cambio Climático (CMNUCC), define en su artículo 1.1 Cambio climático como "un cambio de clima atribuido directa o indirectamente a la actividad humana que altera la composición de la atmósfera mundial y que se suma a la variabilidad natural del clima observada durante períodos de tiempo comparables ${ }^{18}$."

Por su parte, la calidad del aire es más bien un 'fenómeno local', relacionado por la concentración en el aire de determinados contaminantes atmosféricos (partículas, óxidos de azufre (SOx), óxidos de nitrógeno (NOx), ozono troposférico (O3) o monóxido de carbono (CO), entre otros). La Ley 34/2007, de 15 de noviembre, de calidad del aire y protección de la atmósfera en su artículo 3 e) define contaminación atmosférica como "La presencia en la atmósfera de materias, sustancias o formas de energía que impliquen molestia grave, riesgo o daño para la seguridad o la salud de las personas, el medio ambiente y demás bienes de cualquier naturaleza”.

En todo caso, tanto uno como otro concepto hacen referencia a alteraciones atmosféricas producidas por la actividad humana, que los informes del Grupo Intergubernamental de Expertos sobre el Cambio Climático indican que están intensamente relacionadas, hasta el punto de que se prevé un descenso de la calidad el aire en las ciudades en el futuro debido al cambio climático ${ }^{19}$.

\footnotetext{
${ }^{18}$ Sin lugar a dudas, desde un punto de vista estrictamente jurídico esta definición se ofrece como mucho más accesible que la ofrecida por el Panel Intergubernamental del Cambio Climático, conocido por el acrónimo en inglés IPCC que entiende por tal: "Variación del estado del clima identificable (por ejemplo, mediante pruebas estadísticas) en las variaciones del valor medio y/o en la variabilidad de sus propiedades, que persiste durante largos períodos de tiempo, generalmente decenios o períodos más largos. El cambio climático puede deberse a procesos internos naturales o a forzamientos externos tales como modulaciones de los ciclos solares, erupciones volcánicas o cambios antropógenos persistentes de la composición de la atmósfera o del uso del suelo".

Vid. IPCC, 2013: Glosario, en 'Cambio Climático 2013. Bases físicas. Contribución del Grupo de trabajo I al Quinto Informe de Evaluación del Grupo Intergubernamental de Expertos sobre el Cambio Climático'.

19 Calidad del aire y cambio climático, Agencia Europea de Medio Ambiente 2013 $<$ https://www.eea.europa.eu/es/senales/senales-2013/articulos/cambio-climatico-y-aire $>$.
} 


\section{Normativa reguladora de la calidad del aire y la contaminación atmosférica.}

Pues bien, a diferencia de lo que ocurre en materia de cambio climático, la calidad del aire sí es una materia profusamente regulada en nuestro ordenamiento jurídico: la Ley 34/2007, de 15 de noviembre, de Calidad del Aire y Protección de la Atmósfera y los Reales Decretos 100/2011, de 28 de enero, por el que se actualiza el catálogo de actividades potencialmente contaminadoras de la atmósfera y se establecen las disposiciones básicas para su aplicación, 102/2011, de 28 de enero, relativo a la mejora de la calidad del aire y 818/2018, de 6 de julio, sobre medidas para la reducción de las emisiones nacionales de determinados contaminantes atmosféricos. Normativa estatal básica tributaria de la transposición de la normativa comunitaria, la vigente Directiva 2008/50/CE, relativa a la calidad del aire ambiente y a una atmósfera más limpia en Europa y sus antecesoras ${ }^{20}$.

La citada Ley 34/2007 de calidad del aire y protección de la atmósfera parte del reconocimiento de ésta como "bien común indispensable para la vida respecto del cual todas las personas tienen el derecho de su uso y disfrute y la obligación de su conservación" y apunta que "(...) la contaminación atmosférica continúa siendo motivo de seria preocupación en España y en el resto de Europa" ${ }^{21}$. No es para menos, la propia Agencia Europea de Medio Ambiente, en su último informe sobre la calidad del aire en Europa ${ }^{22}$, estimaba que en España fallecen 8.900 personas cada año a causa del dióxido de nitrógeno ${ }^{23}$.

\footnotetext{
${ }^{20}$ Siendo éstas la Directiva 'Madre' 96/62/CE del Consejo de 27 de septiembre de 1996 sobre evaluación y gestión de la calidad del aire ambiente y las Directivas 'Hijas', la 1999/30/CE de 22 de abril relativa a los valores límite de dióxido de azufre, dióxido de nitrógeno y óxidos de nitrógeno, la 2000/69/CE de 16 de noviembre sobre los valores límite para el benceno y el monóxido de carbono en el aire ambiente y la 2002/3/CE de 12 de febrero relativa al ozono en el aire ambiente.

Todas ellas han sido expresamente derogadas (art. 31) y sustituidas por la citada Directiva 2008/50/CE, relativa a la calidad del aire ambiente y a una atmósfera más limpia en Europa.

${ }^{21}$ Preámbulo de la Ley 34/2007 de calidad del aire y protección de la atmósfera.

${ }^{22}$ Disponible en: <https://www.eea.europa.eu/themes/air/country-fact-sheets/spain>.

23 La "muerte prematura debida a la contaminación" se traduce normalmente en un acortamiento de la vida de meses o años.
} 
El objeto de esta Ley es establecer las bases en materia de prevención, vigilancia y reducción de la contaminación atmosférica con el fin de evitar y cuando esto no sea posible, aminorar los daños que de esta puedan derivarse para las personas, el medio ambiente y demás bienes de cualquier naturaleza.

Para ello, se establecen diferentes medidas de evaluación y gestión de la calidad del aire (Capítulo II), se somete a ciertas actividades -las relacionadas en el "Catálogo de actividades potencialmente contaminantes de la atmósfera" de su Anexo IV- a un régimen de intervención administrativa previa (Capítulo III) y se obliga a las distintas administraciones territoriales a la puesta en marcha de planes y programas "para prevenir y reducir la contaminación atmosférica y sus efectos transfronterizos, así como para minimizar sus impactos negativos" -caso de la administración General del Estado- y "para la mejora de la calidad del aire"24, en el caso de Comunidades Autónomas y Entidades locales (Capítulo IV).

La técnica empleada para el control de la calidad del aire es el establecimiento de objetivos de calidad, entendidos éstos como límites de concentración de distintos contaminantes atmosféricos relacionados en el Anexo I de la Ley 34/2007, tales como los óxidos de azufre, nitrógeno o carbono, ozono troposférico, compuestos orgánicos volátiles, etc. cuya superación impone el deber de elaborar planes de acción para la adopción de medidas que garanticen la consecución de los mismos en aras de protección de la salud y la calidad ambiental. Así, el artículo 24 del Real Decreto 102/2011, de 28 de enero, relativo a la mejora de la calidad del aire, señala que "cuando en determinadas zonas o aglomeraciones los niveles de contaminantes en el aire ambiente superen cualquier valor límite o valor objetivo, así como el margen de tolerancia correspondiente a cada caso, las Comunidades Autónomas aprobarán planes de calidad del aire para esas zonas y aglomeraciones con el fin de conseguir respetar el valor límite o el valor objetivo correspondiente..."

\footnotetext{
${ }^{24}$ Artículo 16.2 de la Ley 34/2007, de 15 de noviembre, de Calidad del Aire y Protección de la Atmósfera.
} 
A modo de ejemplo, la Directiva 2008/50/CE y la normativa estatal de transposición, definen como valores límite para el dióxido de nitrógeno (NO2):

- un valor límite anual de $40 \mu \mathrm{g} / \mathrm{m}^{3}$, como media que no debe superar ninguna estación.

- un valor límite horario de $200 \mu \mathrm{g} / \mathrm{m}^{3}$, que no podrá superarse más de 18 veces por año civil.

De superarse estos niveles, las administraciones públicas deben adoptar "planes y programas para la mejora de la calidad del aire y el cumplimiento de los objetivos de calidad del aire en su ámbito territorial, así como para minimizar o evitar los impactos negativos de la contaminación atmosférica" (art. 16 de la Ley 34/2007, de 15 de noviembre, de calidad del aire y protección de la atmósfera).

\section{La creciente litigiosidad en materia de calidad del aire.}

a) Planes y Estrategias de Calidad del Aire.

En materia de calidad del aire sí que existe cierta práctica y experiencia en nuestros Tribunales, que han abordado la validez o suficiencia de determinadas medidas ejecutivas normativa y de planificación en relación con la protección de la calidad del aire.

Un primer ejemplo, ciertamente reseñable por cuanto supone la superación del encorsetamiento de la revisión jurisdiccional de la inactividad de la administración que diseña la Ley reguladora de la jurisdicción contencioso administrativo anteriormente señalado, es la Sentencia 940/2018 de 19 de octubre de la Sección Primera de la Sala de lo Contencioso Administrativo del Tribunal Superior de Justicia de Castilla y León, sede de Valladolid (rec. 672/2016) a instancias de una organización ecologista en la que la Sala acordó "declara[r] la obligación de la Administración Autonómica demandada de elaborar y aprobar los preceptivos Planes de Calidad del Aire para las siguientes zonas: Salamanca, Duero Norte, Duero Sur, Montaña Sur, Valle del Tiétar y Alberche, Sur y Este de Castilla y León" una vez acreditado el incumplimiento de la administración autonómica de adoptar planes y programas 
para la mejora de la calidad del aire y el cumplimiento de los objetivos de calidad del aire en las zonas en las que los niveles de uno o más contaminantes regulados superen dichos objetivos.

En el ámbito madrileño y con motivo de la puesta en marcha del Protocolo de medidas a adoptar durante episodios de alta contaminación de dióxido de nitrógeno, la Sección Segunda de la Sala de lo Contencioso Administrativo del Tribunal Superior de Justicia de Madrid ha tenido ocasión de pronunciarse en sus Sentencias 168/2019 y 177/2019 de 6 de marzo (recs. 139/2017 y 243/2016) y 333 /2019, de 24 de abril (rec. 18/2017), respecto de la validez de este tipo de medidas, señalando que de acuerdo a lo dispuesto en el artículo 16.4 de la citada Ley 34/2007 “(...) los Ayuntamientos pueden legítimamente, en el ámbito de las competencias propias que les reconoce el ordenamiento jurídico, adoptar medidas de restricción del tráfico y circulación de vehículos de motor como las contempladas en el Protocolo objeto de impugnación en el presente recurso, al amparo de las previsiones de las Directivas comunitarias y normas estatales legales y reglamentarias a que acabamos de hacer mención, a las que deben añadirse las normas atributivas a la Administración municipal de un título competencial específico en materia de tráfico, circulación de vehículos a motor y seguridad vial: artículo 25.2.g) de la Ley 7/1985, de 2 de abril, reguladora de las Bases del Régimen Local -que incluye entre las competencias propias de los Municipios las relativas al tráfico, estacionamiento de vehículos y movilidad- y 7 del Real Decreto legislativo 6/2015, de 30 de octubre, por el que se aprueba el Texto Refundido de la Ley sobre Tráfico, Circulación de Vehículos a Motor y Seguridad Vial (que reproduce, en esencia, el tenor del artículo del mismo ordinal del anterior Texto Articulado aprobado por Real Decreto legislativo 339/1990, de 2 de marzo), que asigna a los Municipios competencias en materia de regulación, ordenación, gestión, vigilancia y disciplina del tráfico en las vías urbanas de su titularidad [apartado a)], para el "cierre de vías urbanas cuando sea necesario" [apartado f)] y para la "restricción de la circulación a determinados vehículos en vías urbanas por motivos medioambientales" [apartado g)]".

Y añade que resulta “(...) de destacar que, en todo caso, autoriza de modo específico la adopción de medidas como las aquí combatidas la Ley 34/2007, 
de 15 de noviembre, de Calidad del Aire y Protección de la Atmósfera, que impone, por lo que a las Administraciones locales concierne, a los municipios de más de 100.000 habitantes y aglomeraciones ciertas obligaciones, entre las que, por lo que aquí interesa, destaca tanto la elaboración de planes y programas para los objetivos de calidad del aire, integrando las consideraciones relativas a la protección atmosférica en la planificación de las distintas políticas sectoriales, como la eventual adopción, cuando sea necesario, de medidas de restricción total o parcial del tráfico".

b) La ZBE 'Madrid Central'

También ha sido objeto de numerosos recursos la Ordenanza de Movilidad Sostenible (OMS) de octubre de 2018 del mismo Ayuntamiento de Madrid. Cabe destacar que esta norma municipal supone el marco regulatorio de la Zona de Bajas Emisiones (ZBE) 'Madrid Central', con la finalidad de "a) Reducir los niveles de contaminación ambiental y mejorar la calidad de vida de sus residentes; b) Promover una movilidad más sostenible, favoreciendo el transporte público colectivo y los medios y vehículos menos contaminantes, y c) Disminuir la intensidad de tráfico, recuperando espacio público para el peatón" (art. 22 OMS).

Ante la Sala de lo Contencioso Administrativo (Sección Segunda) del Tribunal Superior de Justicia de Madrid se formularon como decíamos numerosos recursos, en los que actuó como codemandada una organización ecologista en defensa del acierto y legalidad de la OMS. Por parte de las distintas recurrentes (un Grupo Municipal entonces en la oposición, la propia Comunidad de Madrid, varias empresas salva-multas...) se formuló además la solicitud de suspensión cautelar de la eficacia de las concretas previsiones atinentes a la ZBE 'Madrid Central'. Pretensión que no prosperó en ninguno de los recursos por cuanto la Sala consideró que la ZBE se configura como una medida que persigue la protección del medio ambiente y la ordenación de la movilidad para la protección de la seguridad de las personas y la seguridad vial y que supone 
una regulación razonable, proporcional y que responde con objetividad a la consecución del interés general ${ }^{25}$.

Sin embargo, la Sala finalmente declaró la nulidad del artículo 23 OMS, el que precisamente daba cobertura jurídica a la ZBE 'Madrid Central', en virtud de las Sentencias 445/2020 de 27 de julio, rec. 988/2018, 446/2020 de 27 de julio, rec. 902/2018 y 447/2020 de 27 de julio, rec. 911/2018.

Y acordó dicha nulidad por entender insuficiente la Memoria Económica incorporada al expediente administrativo de aprobación de la Ordenanza respecto de los costes económicos que la puesta en marcha de uno sólo de sus artículos podría tener así como por no haberse repetido el trámite de información pública tras haberse introducido una regulación más precisa de "Madrid Central" con posterioridad a la celebración de dicho trámite.

En todo caso, estas sentencias no son firmes, por haber interpuesto contra las mismas recurso de casación la organización ecologista que compareció como codemandada 26 .

Por otra parte, no puede dejar de citarse el conflicto -primero político y posterior judicial- suscitado a raíz del intento de desmantelamiento por la nueva corporación municipal surgida tras las elecciones municipales de 2019 de la Zona de Bajas Emisiones 'Madrid Central'27.

En efecto, a inicios del verano de 2019 se adoptó el Acuerdo de la Junta de Gobierno de la Ciudad de Madrid de 27 de junio de 2019 por el que se establece un periodo de aviso en relación con el acceso a la Zona de Bajas

25 Así lo señalan los Autos de la Sala de lo Contencioso-Administrativo en los que se desestiman las pretensiones de suspensión cautelar de la misma (Autos de 21 de diciembre de 2018 (dictados en los PO 902/2018 y 988/2018) o de 10 de abril de 2019 (PO 1091/2018).

${ }^{26}$ Cosa que no hizo, por cierto, el Ayuntamiento de Madrid.

27 Todo ello sin dejar de mencionar que la Comisión Europea inició formalmente un procedimiento de infracción contra el Reino de España relativo la aplicación de la Directiva 2008/50/CE en España, en particular en lo que se refiere al cumplimiento de los valores establecidos para el dióxido de nitrógeno $\left(\mathrm{NO}_{2}\right)$, en las zonas ES1301 Madrid, ES0901 Área de Barcelona y ES0902 Vallés-Baix Llobregat. Procedimiento que -ante la decidida voluntad de la nueva corporación municipal de Madrid de revertir las medidas previamente acordadas por el Ayuntamiento con la Comisión Europea- desembocó en la decisión del Colegio de Comisarios de 25 de julio de 2019 de demandar a España ante el Tribunal de Justicia de la Unión Europea.

Vid. <https://ec.europa.eu/commission/presscorner/detail/es/IP 19 4256>. 
Emisiones Madrid Central; una suerte de "moratoria"28 en el ejercicio de la potestad sancionadora de la administración local para velar por el cumplimiento de las restricciones de acceso a 'Madrid Central', lo que se consideró como un retroceso en el estándar de protección de la salud pública y el medio ambiente frente a la contaminación atmosférica tanto de partículas como de NOx.

En este litigio, el Juzgado de lo Contencioso Administrativo número 24 de Madrid adoptó la medida cautelarísima inaudita parte de suspensión de la eficacia de tal Acuerdo municipal interesada por la organización ecologista recurrente, Ecologistas en Acción ${ }^{29}$, señalando que "(...) ante una medida dirigida a la protección del medio ambiente como es Madrid Central, el Acuerdo municipal no ofrece ninguna alternativa para suplir la supresión de la zona de bajas emisiones, ni justifica tampoco que la misma haya sido ineficaz o haya producido un daño mayor del que trataba de evitar, ello en el plano medioambiental. La protección a la salud y al medio ambiente son principios que deben regir la actuación de los poderes públicos, y en este caso es exigible en mayor medida dado que se está suprimiendo una actuación tendente a proteger ambos bienes constitucionales, sin ofrecer alternativas ni medidas opcionales".

Este asunto fue finalmente resuelto en virtud de la Sentencia del Juzgado de 17 de junio de 2020, firme por no haber sido recurrida de contrario por el Ayuntamiento de Madrid $^{30}$, estimatoria de la demanda y anulatoria del Acuerdo municipal por entender, entre otras consideraciones, que el mismo infringía el principio de no regresión ambiental al disminuir "el umbral de protección

\footnotetext{
${ }^{28}$ Este Acuerdo de la Junta de Gobierno de la Ciudad de Madrid de 27 de junio de 2019 venía a sustituir el normal funcionamiento y ejercicio de la potestad sancionadora de la administración para el control del cumplimiento de las normas de acceso a la ZBE Madrid Central (que elimina el tránsito de vehículos no residentes que no estén etiquetados como ECO 00 emisiones dentro de su perímetro) por el envío de 'avisos informativos' al infractor.

${ }^{29}$ Auto de 16 de julio de 2019 del Juzgado de lo Contencioso Administrativo núm. 24 de Madrid (rec. 298/2019), F 6ㅇ․

30 Ante el Juzgado 7 se sustanciaron (y acumularon) sendos recursos a instancias de Greenpeace España y el Grupo Municipal Socialista tramitados en el procedimiento 307/2019 en el que se acordó igualmente la medida cautelar suspensiva de su eficacia en virtud del Auto 152/2019 de 19 de julio.
}

Este procedimiento fue resuelto por la Sentencia 103/2020 de 17 de junio igualmente anulatoria del Acuerdo impugnado. 
ambiental en materia de calidad del aire frente a su principal fuente de contaminación consistente en el tráfico rodado urbano".

Contra ese Acuerdo de la Junta de Gobierno municipal de 27 de junio de 2019 se siguió además ante el Juzgado de lo Contencioso-administrativo núm. 27 de Madrid y a instancias del Instituto Internacional de Derecho y Medioambiente y de tres particulares residentes de Madrid Central, el Procedimiento especial de protección de derechos fundamentales núm. 304/2019, aportando así un enfoque diferente, basado en la garantía y protección de los derechos de los afectados por esa pretendida medida reversiva de los efectos de la ZBE 'Madrid Central'. Por Sentencia 198/2020 de 1 de septiembre por entender el juzgador que de la prueba practicada "no puede extraerse, el presupuesto básico de relación causal concreta entre la suspensión y la posible afectación grave a la salud y por ende su conexión con los derechos fundamentales que se alegan vulnerados".

\section{LA LITIGIOSIDAD CLIMÁTICA EN ESPAÑA.}

Son sobradamente conocidas las iniciativas procesales emprendidas en distintos países por organizaciones de la sociedad civil, ecologistas en su mayor parte, en contra de la inactividad de distintos Estados en materia de lucha contra el cambio climático, por entenderse insuficientes o inadecuadas las medidas -de mitigación normalmente- adoptadas por sus Gobiernos frente al alcance del desafío climático y los requerimientos de la comunidad científica; Hablamos de pleitos sobradamente conocidos como el Caso Urgenda c. Holanda, el Caso Leghari c. Pakistán, Juliana c. EE.UU o Natur og Ungdom y Greenpeace Norden c. Gobierno de Noruega.

En España sin embargo, y pese a ser el país más vulnerable al cambio climático de todo el continente europeo ${ }^{31}$, a fecha de hoy no se ha emprendido ningún litigio climático ${ }^{32}$.

\footnotetext{
${ }^{31}$ Agencia Europea del Medio Ambiente. 2017. Climate change impacts and vulnerabilities 2016, disponible en: <http://www.eea.europa.eu/publications/climate-change-impacts-andvulnerability-2016>.

32 Según los informes "El estado del litigio en materia de cambio climático - una revisión global" del Programa de las Naciones Unidas para el Medio Ambiente y el Sabin Center for Climate
} 
Las explicaciones pueden ser varias ${ }^{33}$ :

- La inexistencia de un marco legislativo que establezca obligaciones y compromisos claros y concretos en la lucha contra el cambio climático ${ }^{34,35}$, invocables como motivos de nulidad en sede jurisdiccional,

- La ausencia de reconocimiento constitucional del derecho al medio ambiente adecuado como derecho fundamental, sino como 'principio rector de la vida social y económica ${ }^{36}$,

- La consideración en nuestro ordenamiento jurídico de la potestad reglamentaria como una facultad político-normativa de ejercicio

Change Law de la Universidad de Columbia (2017) y "Global trends in climate change litigation: 2019 snapshot" del Grantham Research Institute on Climate Change (2019), sin embargo, en España se habrían entablado nada menos que 13 litigios climáticos.

Ambos estudios emplean como fuente la Climate Change Litigation Database, del Sabin Center for Climate Change Law, accesible en: <http://climatecasechart.com/>. Acudiendo a ésta, se comprueba como todos y cada uno de estos litigios españoles corresponden a recursos formulados por distintas sociedades industriales contra los créditos de emisiones otorgados por los Planes Nacionales de Asignación, lo que en nuestra opinión no puede reputarse como litigación climática.

${ }^{33}$ Un análisis más profundo de esta cuestión la han llevado a cabo FERNÁNDEZ EGEA, R y SIMOU, S, 2019, ope. cit.

${ }^{34}$ A fecha de hoy, la tantas veces anunciada Ley de Cambio Climático no pasa de ser un proyecto en tramitación actualmente en el Congreso de los Diputados $<$ https://www.congreso.es/public oficiales/L14/CONG/BOCG/A/BOCG-14-A-19-2.PDF>, aprobado por el Consejo de Ministros y su remitido a las Cortes Generales del Estado para su tramitación el pasado 19 de mayo de $2020<$ https://www.miteco.gob.es/es/ministerio/proyectode-ley-de-cambio-climatico-y-transicion-energetica.aspx $>$.

${ }^{35} \mathrm{Ni}$ la Ley $1 / 2005$, de 9 de marzo, por la que se regula el régimen del comercio de derechos de emisión de gases de efecto invernadero, ni la reciente Ley 9/2020, de 16 de diciembre, por la que se modifica la anterior pueden, en puridad, reputarse como acción contra el cambio climático, sino normas reguladoras del intercambio (compraventa) de derechos de emisión.

${ }^{36}$ Por mor de su inclusión sistemática dentro del Capítulo Tercero y no del Segundo, del Título II de la Constitución Española, no invocable por tanto directamente ante los Tribunales ordinarios ni en amparo ante el Tribunal Constitución ex art. 53.2 de la Constitución Española.

En palabras de VELASCO CABALLERO, F: "El «medio ambiente adecuado» presenta una doble manifestación, como derecho subjetivo, en nuestra Constitución. Por un lado, el artículo 45.1 CE proclama, como derecho subjetivo constitucional, aunque no fundamental, el "derecho a disfrutar de un medio ambiente adecuado para el desarrollo de la persona"; se trata de un verdadero derecho público subjetivo, tanto de defensa como prestacional, si bien su protección judicial consiste en el recurso contencioso-administrativo ordinario (artículo 42 LJCA) y no el recurso de amparo ordinario y constitucional.

$Y$, por otro lado, el medio ambiente también forma parte de alguno de los clásicos derechos de libertad a que hace referencia el capítulo II del título I de la Constitución". 
discrecional y la difícil -que no imposible-admisibilidad de la sustitución judicial de la inactividad u omisión administrativa reglamentaria ${ }^{37}$.

- La restrictiva regulación del recurso contra la inactividad de la Administración de los artículos 25.2 y 29.1 de la Ley 29/1998, de 13 de julio, reguladora de la Jurisdicción Contencioso-administrativa que permite tal actuación sólo en caso de que "la Administración, en virtud de una disposición general que no precise de actos de aplicación o en virtud de un acto, contrato o convenio administrativo, esté obligada a realizar una prestación concreta en favor de una o varias personas determinadas ${ }^{38 ",}$ así como

- La inestabilidad política de los últimos años (no en vano, desde diciembre de 2012 se han sucedido 5 legislaturas) y la ausencia por tanto de un Gobierno estable interlocutor de las demandas de lucha contra el cambio climático e impulsor de las medidas legislativas y de planificación que la magnitud del desafío climático exige.

Pese a este marco procesal y normativo tan poco favorable, el pasado 24 de enero de 2020 Greenpeace España, Oxfam Intermón y Ecologistas en Acción interpusieron ante el Consejo de Ministros y Ministras y el Ministerio para la Transición Ecológica y el Reto Demográfico una reclamación previa al amparo de los artículos 22 de la Ley 27/2006, de 18 de julio, por la que se regulan los derechos de acceso a la información, de participación pública y de acceso a la justicia en materia de medio ambiente y 29.1 de la Ley 29/1998, de 13 de julio, reguladora de la Jurisdicción Contencioso-administrativa por no haber

37 Vid. artículo 71.2 de la Ley 29/1998, de 13 de julio, reguladora de la Jurisdicción Contencioso-administrativa y, por todas, las Sentencias de la Sala III del Tribunal Supremo de 7 de octubre de 2002 (Sección Cuarta) o de 30 de mayo de 2003 (Sección Quinta).

38 Ciertamente, este óbice procesal puede ser evitado en virtud de una interpretación sistemática del artículo 22 de la Ley 27/2006, de 18 de julio, por la que se regulan los derechos de acceso a la información, de participación pública y de acceso a la justicia en materia de medio ambiente, que permite a las ONG ambientalistas cualificadas (que cumplan con los requisitos de finalidad, actividad, territorialidad y antigüedad que establece el art. 23 del mismo texto legal) recurrir "Los actos y, en su caso, las omisiones imputables a las autoridades públicas que vulneren las normas relacionadas con el medio ambiente(...)".

A este respecto interesa destacar que dicho precepto fue dictado "al amparo del artículo 149.1. 18. ${ }^{a}$ de la Constitución y en lo relativo a recursos en vía contencioso-administrativa al amparo del artículo 149.1. 6. ${ }^{a}$ de la Constitución" por lo que debe ser entendida como una verdadera norma procesal con carácter de lex especialis respecto del régimen general de la Ley reguladora de la jurisdicción contencioso-administrativa. 
aprobado en plazo el PNIEC (Plan Nacional Integrado de Energía y Clima 2021-2030) infringiendo el Reglamento comunitario de Gobernanza Climática y reclamando la mayor de las ambiciones climáticas y la debida garantía de los derechos humanos y al medio ambiente adecuada de las generaciones presente y futuras ${ }^{39}$.

El petitum de dicha reclamación fue el siguiente:

SOLICITAMOS que, teniendo por presentado el presente escrito junto con la documentación que se acompaña, se sirva admitirlo y, en su virtud, tenga por formulada RECLAMACIÓN PREVIA CONTRA LA INACTIVIDAD CLIMÁTICA denunciada $y$, por las razones expuestas, adopte las medidas pertinentes para cumplir las obligaciones históricas y ambientales del Estado Español y así:

- Se aprueben tanto el Plan Nacional Integrado de Energía y Clima de España para el periodo 2021-2030, como la Estrategia a Largo Plazo, con un elevado nivel de protección ambiental, una amplia participación pública y una adecuada evaluación.

- Se establezcan unos objetivos de reducción de gases de efecto invernadero acordes con los compromisos asumidos con la ratificación del Acuerdo de París y las recomendaciones científicas del IPCC para no superar $1,5^{\circ} \mathrm{C}$, en ningún caso inferiores al $55 \%$ en 2030 respecto a 1990 y el cero neto de las emisiones en 2040.

- Se garanticen a este respecto los derechos humanos y al medio ambiente adecuado de las generaciones presentes y futuras, de acuerdo con los principios de solidaridad, sostenibilidad, comunes pero diferenciadas responsabilidades, precaución y buen gobierno consagrados en nuestro ordenamiento jurídico.

Si bien no es objeto de la presente nota el análisis de este primer litigio climático, si procede dejar apuntado que esa reclamación previa derivó

\footnotetext{
${ }^{39}$ Así lo ha anunciado Greenpeace en un Comunicado criticando duramente el Acuerdo del Consejo de Ministros de 31 de marzo de 2020 por el que se autoriza al Ministerio para la Transición Ecológica y el Reto Demográfico a comunicar a la Comisión Europea el Plan Nacional Integrado de Energía y Clima 2021-2030, cuando ese Plan (conocido como PNIEC por sus siglas) se encuentra en periodo de información pública.
}

Vid. $\quad<$ https://es.greenpeace.org/es/sala-de-prensa/comunicados/greenpeace-advierte-que-laprecipitada-presentacion-del-pniec-elude-la-obligacion-del-gobierno-de-garantizar-laparticipacion-publica/>. 
finalmente en la interposición del pertinente recurso contencioso administrativo ante el Tribunal Supremo ${ }^{40}$, que fue admitido a trámite en virtud de Diligencia de Ordenación de 30 de septiembre de 2020. Tras los trámites de rigor, el pasado 15 de diciembre las organizaciones recurrentes presentaban el escrito de demanda ${ }^{41}$.

Se trata de un litigio que vehicula la pretensión de que se establezca un objetivo de reducción de las emisiones de gases de efecto invernadero del 55\% para 2030 a través de la inactividad reglamentaria (climática, por razón de la materia) al no haberse aprobado aún el Plan Nacional Integrado de Energía y Clima al que obligaba el Reglamento (UE) 2018/1999 del Parlamento Europeo y del Consejo de 11 de diciembre de 2018, sobre la gobernanza de la Unión de la Energía y de la Acción por el Clima para "a más tardar el 31 de diciembre de 2019" (artículo 3.1).

Como desarrollan en la demanda las organizaciones recurrentes, el objetivo del $55 \%$ se establece de conformidad con lo señalado por el Programa de Naciones Unidas para el Medio Ambiente su informe de 2019 sobre la disparidad en las emisiones ${ }^{42}$, que estableció en un 7,6\% cada año entre 2020 y 2020 la reducción necesaria de emisiones para poder limitar el calentamiento global a $1,5{ }^{\circ} \mathrm{C}$, y atendiendo a la responsabilidad histórica de España, su capacidad económica y su especial vulnerabilidad a los efectos y consecuencias del cambio climático.

\section{A MODO DE CONCLUSIÓN Y PERSPECTIVAS DE FUTURO}

El litigio climático está ganando una creciente relevancia a nivel internacional como un instrumento ciudadano para el impulso y exigencia de medidas eficaces en la lucha contra el cambio climático.

\footnotetext{
${ }^{40}$ Vid. https://elpais.com/sociedad/2020-09-15/primer-litigio-climatico-en-espana-las-ong-pidenante-el-supremo-mayores-recortes-de-emisiones-al-gobierno.html>.

41 Disponible en: https://www.ecologistasenaccion.org/158818/presentada-ante-el-tribunalsupremo-la-demanda-contra-el-gobierno-por-falta-de-accion-climatica.

${ }^{42}$ Disponible en

$<$ https://wedocs.unep.org/bitstream/handle/20.500.11822/30798/EGR19ESSP.pdf>
} 
Y es que, como bien señala SORO, "cuando ni el legislador ni la Administración dan la talla, quizá sea útil dar la batalla ante los Tribunales"3". Por ello en nuestro país, animados por las iniciativas judiciales emprendidas en otros países -en algunos casos con notables éxitos-, impelidos por la emergencia climática a la que tan vulnerable es nuestro país y con la creciente experiencia en litigiosidad en materia de contaminación atmosférica y calidad del aire, la sociedad civil ha entablado recientemente ante el Tribunal Supremo el primer litigio climático. Se trata de un particular pleito estratégico aprovechando la ventana de oportunidad que brindó la falta de aprobación del Plan Nacional Integrado de Energía y Clima y enfocado desde la perspectiva de la mejor evidencia científica y la garantía de los derechos humanos para, a modo de punta de lanza, implicar a los tribunales también en la lucha contra el cambio climático, como instituciones del Estado que en definitiva son.

Del devenir de este litigio, y de los que vendrán, habrá ocasión de seguir escribiendo.

\section{BIBLIOGRAFIA}

AGENCIA EUROPEA DE MEDIO AMBIENTE, Calidad del aire y cambio climático, 2013.

AGENCIA EUROPEA DEL MEDIO AMBIENTE, Climate change impacts and vulnerabilities 2016, 2017.

BORÁS PENTINAT, SUSANA, "La justicia climática: entre la tutela y la fiscalización de las responsabilidades" en Anuario Mexicano de Derecho Internacional, vol. XIII, 2013.

BURGER, MICHAEL y GUNDLACH, JUSTIN "El estado del litigio en materia de cambio climático - una revisión global", Programa de las Naciones Unidas para el Medio Ambiente y Sabin Center for Climate Change Law de la Universidad de Columbia, Estados Unidos de América, mayo 2017.

43 SORO MATEO, BLANCA "Marco jurídico general de la cuestión climática. Algunas reflexiones a la espera de la aprobación de la ley española de cambio climático y transición energética” en 'El derecho ante el reto del cambio climático', Aranzadi 2020. 
FELIPE PÉREZ, BEATRIZ, e IGLESIAS MÁRQUEZ, DANIEL, "Anhelando justicia en la era del cambio climático: de la teoría a la práctica" en Revista Catalana de Dret Ambiental Vol. IX núm.2 (2018).

FERNÁNDEZ EGEA, ROSA y SIMOU, SOFIA, "Litigación climática en España: posibilidades y límites" en Revista de Derecho Urbanístico y Medio Ambiente núm. 328, marzo 2019

GIL DELGADO, EDUARDO, Cambio climático; Amenazas y soluciones, EGIDEL 2015.

JARÍA-MANZANO, JORDI "La litigació climática a Espanya: una prospectiva"en Revista Catalana de Dret Ambiental Vol. IX núm.2 (2018).

KNOX, JOHN "Cambio climático y derechos humanos", Observatorio Social de la Caixa, septiembre de 2016, disponible en:

$<$ https://observatoriosociallacaixa.org/-/cambio-climatico-y-derechos-humanos $>$ LOPERENA ROTA, DEMETRIO (Coord.), La calidad del aire y la protección de la atmósfera, Thomson Reuters Aranzadi, 2010.

MANUEL MORENO, ÁNGEL "Perspectivas y desarrollos recientes en el Derecho del cambio climático", en A. García Ureta (Dir.) y M.C. Bolaño Piñeiro (Coord.), Nuevas perspectivas del Derecho ambiental en el siglo XXI, Marcial Pons, 2018.

PNUMA, "Informe sobre la disparidad en las emisiones de 2019".

RICH, NATHANIEL, Perdiendo la Tierra, Capitán Swing 2020.

SABIN CENTER FOR CLIMATE CHANGE LAW, "Climate Change Litigation Databases", disponible en < http://climatecasechart.com>.

SALAZAR ORTUÑO, EDUARDO, "El acceso a la justicia ambiental, una cuestión pendiente del Convenio de Aarhus", 2019, CGAE, disponible en $<$ https://www2.abogacia.es/publicaciones/blogs/blog-derecho-ambiental/elacceso-a-la-justicia-ambiental-una-cuestion-pendiente-del-convenio-deaaerhus/>

SALAZAR ORTUÑO, EDUARDO, "El acceso a la justicia ambiental a partir del Convenio de Aarhus", Thomson Reuters Aranzadi, 2019. 
SANCHÍS MORENO, FE "Convenio de Aarhus; Guía sobre el Acceso a la Justicia Ambiental', Consejo General de la Abogacía Española-Asociación para la justicia ambiental (ELAW España), 2007.

SANTAMARÍA ARINAS, René Javier, "La Ley de calidad del aire y protección de la atmósfera diez años después de su entrada en vigor", Revista de Derecho Urbanístico y Medio Ambiente, núm. 325, 2018.

SETZER, JOANA y BYRNES, REBECCA, "Global trends in climate change litigation: 2019 snapshot", 2019. Grantham Research Institute on Climate Change and the Environment and Centre for Climate Change Economics and Policy, London School of Economics and Political Science.

SORO MATEO, BLANCA, "Marco jurídico general de la cuestión climática. Algunas reflexiones a la espera de la aprobación de la ley española de cambio climático y transición energética” en F. L. Hernández González (Dir), El derecho ante el reto del cambio climático, Aranzadi 2020.

THE INTERGOVERNMENTAL PANEL ON CLIMATE CHANGE (IPCC), Cambio Climático 2013. Bases físicas. Contribución del Grupo de trabajo I al Quinto Informe de Evaluación del Grupo Intergubernamental de Expertos sobre el Cambio Climático, 2013.

VELASCO CABALLERO, FRANCISCO, "La protección del Medio Ambiente ante el Tribunal Europeo de Derechos Humanos", en Revista Española de Derecho Constitucional, núm. 45 (1995).

VALENCIA MARTÍN, GERMÁN, "Jurisprudencia constitucional y medio ambiente", Thomson Reuters Aranzadi, 2017.

VILASECA BOIXAREU, ISABEL y SERRA CALVÓ, JORDI, "Litigación climática y separación de poderes", en Revista Catalana de Dret Ambiental Vol. IX núm.2 (2018). 